\title{
«Spital. Illegal. Normal?»: Werden in der Schweiz die Arbeitszeiten der Ärzte tatsächlich respektiert?
}

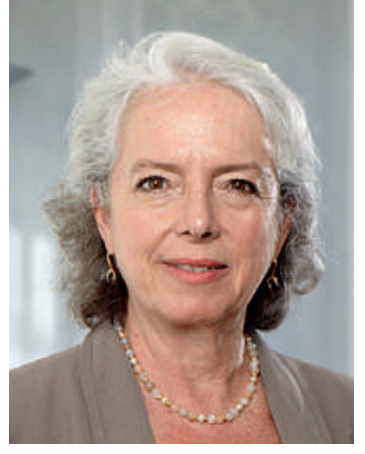

Anfang März hat der VSAO, der Verband Schweizerischer Assistenz- und Oberärztinnen und -ärzte, unter dem Titel «Spital. Illegal. Normal?» eine Kampagne gestartet, um die Öffentlichkeit für die Arbeitsbedingungen der Ärztinnen und Ärzte in den Spitälern zu sensibilisieren [1]. Zwar hat die seit 1. Januar 2005 geltende Unterstellung unter das Arbeitsgesetz dazu geführt, dass ihre Arbeitsbedingungen besser kontrolliert werden und die Spitäler ca. 25\% mehr Assistenzärztinnen und -ärzte einstellen mussten.

Dennoch werden die Arbeitszeiten der Spitalärztinnen und -ärzte sowohl im Hinblick auf die tägliche Arbeitszeit als auch auf die Anzahl an Arbeitstagen weiterhin regelmässig überschritten.

Die zahlreichen vom VSAO zusammengestellten Aussagen belegen, dass die Arbeitsbedingungen der Ärzte nach wie vor nicht immer wahrheitsgemäss dokumentiert werden und dass nur in Ausnahmefällen eine Beschwerde oder Anzeige eingereicht wird. Solche Aussagen kommen aus allen Regionen der Schweiz und sämtlichen Kantonen.

Lediglich der Kanton Bern hat im Jahr 2010 eine Untersuchung der Arbeitsbedingungen in 28 Akutspitälern, 6 psychiatrischen Kliniken und 6 Rehabilitationskliniken in Auftrag gegeben, die belegte, dass es überall und auf allen Ebenen zu Überschreitungen kam, bei der Ärzteschaft aber stärker als beim übrigen Pflegepersonal [2].

\section{Die Ärztinnen müssen als Erste auf bestimmte Wunschvorstellungen verzichten.}

Kann man die Auswirkungen dieser Überlastung messen? Die von der Kampagne aufgeworfene Frage nach der Patientensicherheit hat in der Schweizerischen Ärztezeitung eine kleine Kontroverse ausgelöst [3]: Gibt es wirklich Auswirkungen auf die Qualität der Behandlung? Selbst wenn es offensichtlich ist, dass Müdigkeit die Anzahl an Ausrutschern oder Fehlern erhöht, sind die Ärztinnen und Ärzte doch robust und werden stets ihr eigenes Wohlbefinden zurückstellen. Im Vergleich zu anderen europäischen Ländern mag die Situation in der Schweiz komfortabler erscheinen, doch wenn man mit jungen Schweizer Berufskollegen spricht, schildern sie trotz allem sehr schwierige Arbeitsbedingungen. Ärztin- nen und Ärzte, deren Kinder vor der Berufswahl stehen, raten diesen offen davon ab, den gleichen Berufsweg wie sie einzuschlagen, was zweifellos beunruhigend ist.

Eine 2010 von der Universität Zürich (Buddeberg-Fischer et al.) durchgeführte Kohortenstudie unter 579 seit sieben Jahren in den Kantonen Basel, Bern und Zürich tätigen Ärztinnen und Ärzten zeigt einige interessante Aspekte auf [4]:

Im Vergleich zu ihren männlichen Kollegen müssen Ärztinnen als Erste auf bestimmte Wunschvorstellungen verzichten, insbesondere dann, wenn sie Kinder haben: Sie nehmen Auszeiten und sehen sich nach weniger anspruchsvollen Fachbereichen oder Teilzeitstellen um, lassen sich schneller nieder oder arbeiten in nichtuniversitären Kliniken. Aber es sind nicht nur Frauen, sondern auch immer mehr Männer, die ihre Berufswahl an einer zufriedenstellenden Lebensqualität ausrichten.

Die 100 Mütter der Zürcher Gruppe weisen einen Beschäftigungsgrad von $64 \%$ auf, während er bei den 110 Vätern bei $95 \%$ liegt ... Die Ärztinnen und Ärzte sehen sich in einem Spannungsfeld zwischen Karriere und Familie: Die Familiengründung geht zulasten der Arbeit. Es ist eine horizontale und vertikale Trennung zu beobachten: Männer entscheiden sich für angesehenere Fachbereiche wie Chirurgie oder Kardiologie; in den höheren Ebenen von Wissenschaft und Spitälern finden sich immer weniger Frauen.

In den kommenden Jahren steht die Medizin vor einer enormen Herausforderung, sobald die Alterspyramide schmaler wird [5]. Wir müssen unbedingt kreative Arbeitszeitsmodelle entwickeln, mit den sich die familiären Bedürfnisse vereinbaren lassen, wenn wir nicht noch mehr Arbeitskraft verlieren wollen, vor allem unter den Frauen. Es ist deshalb mehr denn je notwendig, auf die Arbeitszeiten der Spitalärztinnen und -ärzte zu achten.

Monique Gauthey, Fachärztin FMH, Mitglied des Zentralvorstands, Leitung des Ressorts «Spitalärztinnen und -ärzte»

\section{Referenzen}

1 www.spital-illegal.ch

2 Bericht über die Kontrollen in den Spitälern und Kliniken des Kantons Bern 2010/12. www.be.ch/portal/de/index/ mediencenter/medienmitteilungen.meldungNeu.html/ portal/de/meldungen/mm/2013/04/20130403_1520_ gesund_im_spitalfuehrtzuverbesserungen

3 Briefe an die SÄZ, Max Giger. www.saez.ch/docs/saez/2013/16/ de/SAEZ-01450.pdf

4 Buddeberg-Fischer B. The impact of gender and parenthood on physicians' careers - professional and personal situation seven years after graduation. BMC Health Services Research. 2010;10:40.

5 Strategie gegen Ärztemangel und zur Förderung der Hausarztmedizin. Bericht des Bundesrates. 